Periodica Polytechnica Chemical Engineering, 63(2), pp. 270-275, 2019

\title{
The Latent Heat of Supercritical Fluids
}

\author{
Daniel T. Banuti ${ }^{1 *}$ \\ 1 Department of Mechanical and Civil Engineering, California Institute of Technology, \\ 1200 East California Boulevard, Pasadena, CA 91125, USA \\ *Corresponding author, e-mail: dbanuti@caltech.edu, daniel@banuti.com
}

Received: 17 July 2018, Accepted: 15 November 2018, Published online: 21 January 2019

\begin{abstract}
The article discusses the notion of a supercritical latent heat during 'pseudoboiling': Experimental, numerical, and theoretical evidence show that the supercritical state space is not homogeneous, but can be divided into liquid-like and gas-like domains, separated by an extension to the coexistence line -- the Widom line. The key concept are two limit states of ideal liquid and ideal gas, characterized by constant heat capacities, and analyze the transition between them. Then, analogous to subcritical vaporization, a supercritical state transition from liquid to gaseous overcomes intermolecular attractive forces, albeit over a finite temperature interval rather than at an equilibrium temperature. This distributed latent heat is in fact approximately invariant with respect to pressure for $\left(0<p<3 p_{c r}\right)$ and is thus valid at subcritical and supercritical conditions. This view also changes the perspective on subcritical latent heat: while it is an accurate representation of the required energy at very low pressures, the contribution of the distributed latent heat dominates the equilibrium latent heat as the critical pressure is approached.
\end{abstract}

\section{Keywords}

pseudoboiling, Widom line, supercritical, transcritical, phase transition

\section{Introduction}

The supercritical state space has long been regarded as a continuous domain, in which the differences between liquids and gases vanish. However, experimental [1-6], numerical [7, 8], and theoretical [9, 10] advances have shown that distinct supercritical liquid-like and gas-like regions can in fact be distinguished, separated by the Widom line (WL) $[4,5,11,12]$, an extension to the coexistence line as illustrated in Fig. 1. Many definitions of the Widom line, typically as extrema of the thermodynamic response functions are used; here, WL refers to the locus of supercritical states with a maximum isobaric specific heat capacity.

WL of the liquid-vapor critical point are a general property of pure fluids; their slope depends on the fluid acentric factor [13]. Only the WL of simple fluids map according to the corresponding states principle; a wide range of fluids adheres to the extended corresponding states principle [13]. The state space of mixtures depends on the van Konynenburg and Scott [14] classification of the involved components [15]: Mixtures of similar fluids (Type I) exhibit a single common WL, while mixtures of dissimilar fluids (Type III) may develop several WL, corresponding to the mixture components.

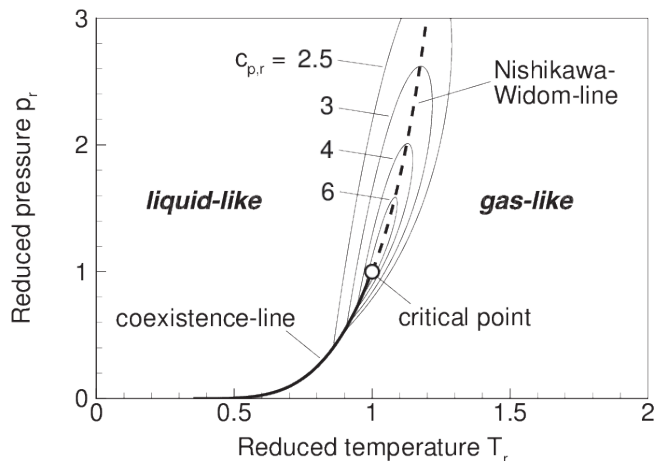

Fig. 1 Structure of the fluid state space around the critical point $\left(T_{c r}, p_{c r}\right)$. The supercritical domain can be further divided into a liquidlike and a gas-like domain, separated by the Widom line (dashed). $c_{p, r}$ is the isobaric heat capacity nondimensionalized with its ideal gas value.

The existence of liquid-like and gas-like states at supercritical pressures suggests the question as to whether a supercritical state transition akin to subcritical vaporization exists. Similarities have been observed in heat transfer, where oscillations that can be attributed to boiling at subcritical pressure are also observed at moderate supercritical pressure $[16,17]$ - so-called 'pseudoboiling'. Pseudoboiling is associated with the maxima in specific heat capacity 
and thermal expansion upon crossing the WL, causing a delayed temperature increase and strong expansion when heat is added, similarly to subcritical boiling [18].

The energetical characteristics of the transition remain unclear. While there is no supercritical equilibrium state with liquid and gaseous phases in coexistence, intermolecular attraction cannot be expected to simply vanish by increasing the fluid pressure beyond the critical point.

Thus, the purpose of this article is to explore similarities between liquid and liquid-like to gas and gas-like transitions at subcritical and supercritical pressure.

\section{Results}

The approach of this article is theoretical, based on classical chemical engineering approaches [19]. Reference fluid data are taken from the NIST database [20] for argon.

The analysis starts by regarding the isobars of the specific enthalpy of pure argon shown in Fig. 2, where the zero of the enthalpy scale is set at $T=0 \mathrm{~K}$ and zero density. The subcritical isobars show the discontinuous change from liquid to gaseous upon reaching the saturation temperature, while all discontinuities vanish at the critical pressure $p_{c r}$ and critical temperature $T_{c r}$. It is convenient to express states nondimensionalized with the critical parameters, e.g. in terms of their reduced pressure $p_{r}=p / p_{c r}$ and reduced temperature $T_{r}=T / T_{c r}$.

\subsection{Pseudoboiling and distributed latent heat}

However, Fig. 2 also shows that supercritical isobars exhibit a region in which the specific enthalpy increases disproportionally fast over a small but finite temperature interval, sometimes called 'pseudoboiling' (PB) [21, 18]. The resemblance with subcritical vaporization is most striking for the critical isobar, but the transition can still be visually identified for higher pressures. The peak of the PB transition [18], at which the isobaric specific heat capacity $c_{p}$ is maximal, coincides with the WL traced by the gray dashed line in Fig. 2.

Towards low temperatures, the liquid and liquid-like specific enthalpies approach a limit which can be approximated as a linear function with

$$
h_{i L}=h_{0, L}+c_{p, i L} T \text {. }
$$

with a reference specific enthalpy $h_{0, L}$ and the constant ideal liquid heat capacity $c_{p, i L}$. Similarly, the specific enthalpy of a perfect gas is

$$
h_{i G}=c_{p, i G} T \text {, }
$$

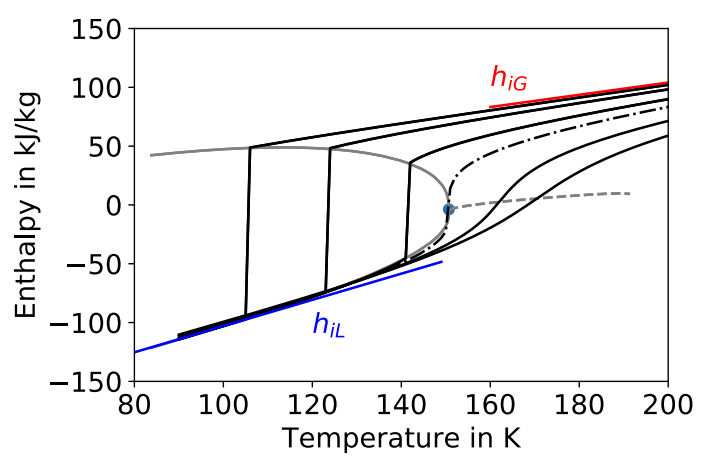

Fig. 2 Specific enthalpy of argon as a function of temperature for (from left to right) subcritical ( $\left.p_{r}=\{0.1,0.3,0.7\}\right)$, critical (dash-dotted), and supercritical pressures $\left(p_{r}=\{1.5,2.0\}\right)$. Gray solid lines are the saturation enthalpies, gray dashes mark the Widom line. Linear asymptotes are the ideal liquid enthalpy $h_{i L}$ (blue) and ideal gas enthalpy $h_{i G}$ (red).

which is approached towards high temperatures. While the constant isobaric specific heat capacity of the ideal gas $c_{p, i G}$ is a result of kinetic theory, the assumption of a constant liquid heat capacity is merely an approximation; in reality, the liquid heat capacity changes even far away from the critical point, albeit slowly.

Along $h_{i l}$, only variations in temperature have an effect on liquid and liquid-like specific enthalpies. Moreover, $h_{i l}$ approximates both saturated states at low pressures $\left(p_{r}<0.2\right)$ and bulk liquid states away from the coexistence line, c.f. Fig. 1. With two state variables fixed, $T$ and $h_{i l}$, the thermodynamic state of a pure fluid in a single phase is uniquely determined. As the enthalpy departure from ideal conditions is caused by intermolecular interactions, an identical departure is assumed to reflect a similarity in molecular structure.

Then, states along $h_{i l}$ share a similar densely packed liquid or liquid-like structure, whereas states along $h_{i G}$ share the ideal gas structure of isolated molecules. A transition from the densely packed liquid state to the perfect gas state will require energy to overcome intermolecular forces, at subcritical and supercritical pressures. This is illustrated in Fig. 3, which shows the molecular break-up of a liquid during isobaric heating from a liquid state $\left(T_{r}<0.7\right)$ to an ideal gas state $(\operatorname{Tr} \gg 1)$. The transitional state is the multiphase regime at subcritical pressure, and an intermediate state while crossing the WL at supercritical pressure. Thus, isobaric heating processes from liquid and liquid-like states to the sub- and supercritical perfect gas states are identical from the molecular perspective. Energy is required to overcome intermolecular attractive forces regardless of pressure.

In this sense, one can understand the required transition energy at supercritical pressures as a distributed latent 


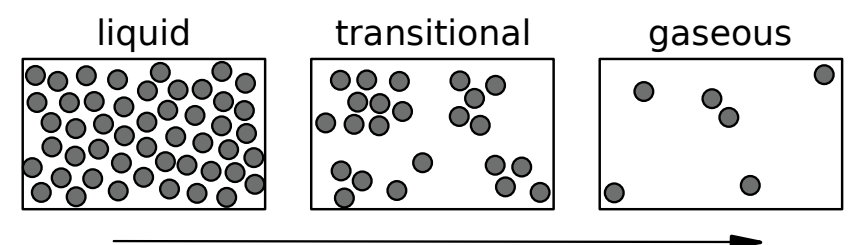

Fig. 3 Molecular structure between liquid and ideal gas limit states at subcritical and moderately supercritical pressures. During the transition, energy addition is required to overcome intermolecular forces and to transform the molecular structure from the densely packed liquid to the dilute gas.

heat that needs to be supplied over a finite temperature interval, rather than at an equilibrium coexistence condition observed at subcritical vaporization. The ideal gas limiting state can be reached for reduced pressures $p_{r} \leq 3$ [18], at higher pressures this similarity breaks down.

Closer inspection of Fig. 2, however, reveals that instances of such a distributed latent heat can also be observed in subcritical transitions. While the transition from the ideal liquid to the ideal gas limit states occurs instantaneous at very low pressures $p_{r} \ll 1$, the difference increases as the critical point is approached. Take the isobar $p_{r}=0.7$ in Fig. 2 for example, with a saturation temperature of $\sim 140 \mathrm{~K}$. Only about two thirds of the transition enthalpy from $h_{i l}$ to $h_{i G}$ occur at the constant equilibrium saturation temperature. An offset $\delta h_{\text {liquid }}(T)$ is found between the saturated liquid specific enthalpy $h_{L, s a t}(T)$ and the liquid limit $h_{i L}(T)$

$\delta h_{\text {liquid }}(T)=h_{L, \text { sat }}(T)-h_{i L}(T)$,

and, likewise, between the saturated vapor specific enthalpy $h_{V, \text { sat }}(T)$ and the ideal gas limit $h_{i G}(T)$

$\delta h_{\text {gas }}(T)=h_{i G}(T)-h_{V, \text { sat }}(T)$.

The larger portion of the total required specific enthalpy is supplied in the vapor after vaporization.
Figure 4 illustrates this distributed latent heat in terms of the isobaric specific heat capacity. At very low pressures, all of the vaporization energy is supplied in a Dirac delta at the saturation temperature. Ideal liquid and gaseous conditions are attained immediately prior to and after the transition. With increasing pressure, deviations from the ideal heat capacities emerge on the both the liquid and the vapor side of the coexistence line, c.f. Fig. 1: the transition is distributed over a finite temperature interval containing the saturation state at which the heat capacity diverges. When the critical pressure is exceeded, the Dirac-like contribution vanishes; all of the required transition energy is supplied over a finite temperature interval without any equilibrium state. The temperature increase upon heat addition merely slows down when crossing the WL.

The linear approximations of the ideal limit states allow to calculate the distributed latent heat $\delta h_{\text {dist }}$ required to transition between the ideal liquid and the ideal gaseous state at a specific temperature,

$\delta h_{\text {dist }}=\delta h_{L}+\delta h_{\text {gas }}+\delta h_{\text {liquid }}$

with the classical (equilibrium) latent heat $\delta h_{L}=$ $h_{V, \text { sat }}-h_{L, \text { sat }}$, and the contributions of the real liquid $\delta h_{\text {liquid }}$ and the real gas $\delta h_{\text {gas }}$ contributions. This can be cast in terms of the linear approximations for $h_{i l}$ and $h_{i G}$ as

$\delta h_{d i s t}=h_{0, L}+\left(c_{p i G}-c_{p, i L}\right) T$.

Figure 2 shows that higher pressures result in higher discrepancies between the equilibrium latent heat and the distributed latent heat. The relation Eq. (5) is evaluated in Fig. 5 as a function of temperature. Towards the triple point, the phase transition completely occurs under equilibrium conditions, i.e. $\delta h_{L}=\delta h_{\text {dist }}$. The deviations are more pronounced on the vapor side; generally, at a given temperature, $\delta h_{\text {gas }}>\delta h_{\text {liquid }}$. At $T \sim 145 \mathrm{~K},\left(T_{r}=0.96\right)$

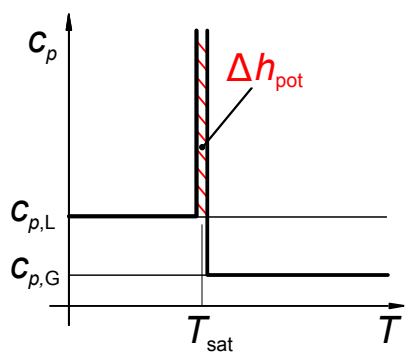

low pressure subcritical

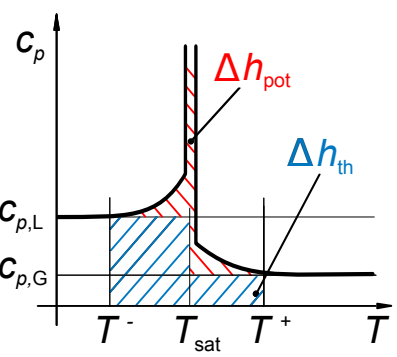

high pressure subcritical

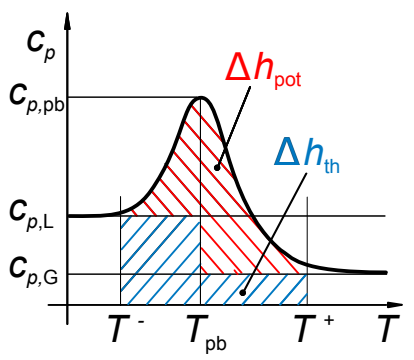

supercritical

Fig. 4 Isobaric specific heat capacity in the vicinity of the liquid to gas transition at low and high subcritical and at supercritical pressure. The transition region expands to finite temperature intervals with increasing pressure. Red: energy required to overcome the potential energy between the molecules; Blue: energy contribution to increasing the temperature of the fluid. 


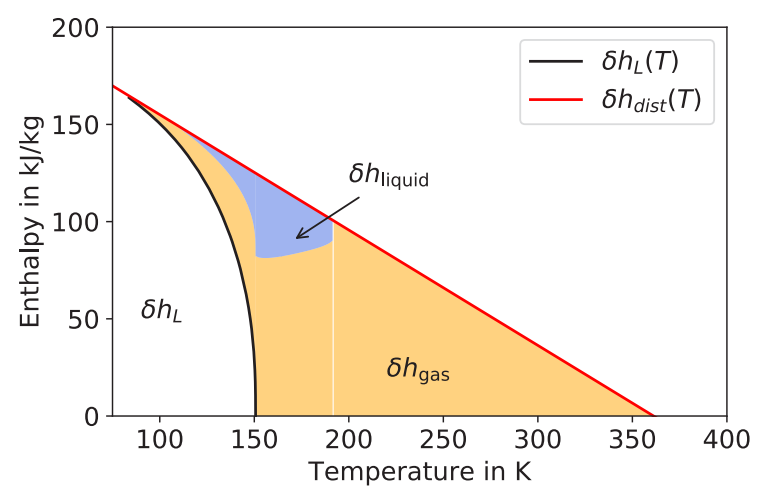

Fig. 5 Equilibrium latent heat and distributed latent heat contributions. The required energy to transition from the ideal liquid to the ideal gas at a temperature $T$ is split in three contributions of equilibrium latent heat $\delta h_{L}$, the ideal liquid to real liquid $\delta h_{\text {liquid }}$ transition, and the ideal gas to real vapor transition $\delta h_{\text {gas }}$, calculated from Eqs. (3)-(6).

the contribution of the nonequlibrium heat addition exceeds the equilibrium latent heat.

Beyond the critical point, $\delta h_{L}=0$, and all contributions are from the distributed parts. With the WL approaching the ideal liquid limit, the liquid contribution in Fig. 5 vanishes at $\sim 200 \mathrm{~K}$.

\subsection{Caloric equivalence between subcritical and supercritical transitions}

So far, the discussion focused on the different energetic contributions necessary to transform a closely packed liquid to a dilute gas in which molecules do no longer interact with each other. However, there are also similarities between subcritical and supercritical transitions between liquid and liquid-like states, to gaseous and gas-like states.

The ideal limits of an incompressible liquid and an ideal gas share an important characteristic: enthalpies in both are assumed to be essentially pressure-independent. This implies an interesting corollary: when the end points of a heating process are pressure-independent, then, every process between both states is likewise pressure-independent. Specifically, there is no difference between subcritical and supercritical transitions.

Figure 6 illustrates this. All shown isobars with reduced pressures between 0.1 and 3.0 converge towards the ideal liquid limit $h_{i L}(T)$ at low temperatures and towards the ideal gas limit $h_{i G}(T)$ at higher temperatures. For example, a transition from a liquid at $T=100 \mathrm{~K}$ to a gas at $T>500 \mathrm{~K}$ requires approximately the same increase in specific enthalpy for all isobars shown.

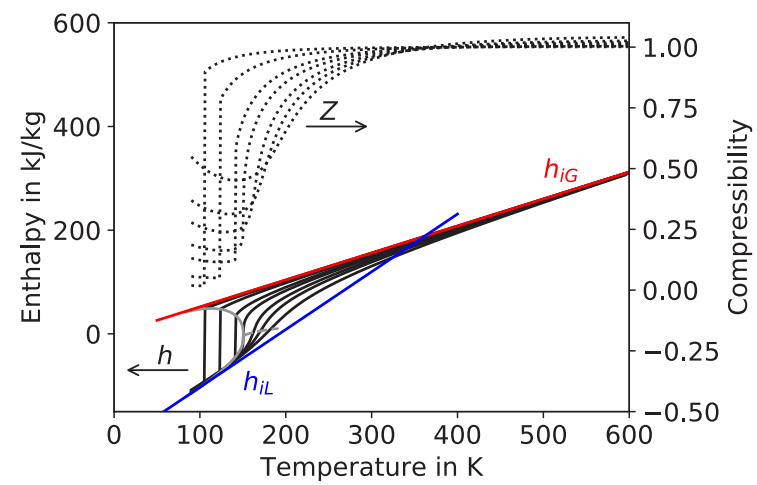

Fig. 6 Specific enthalpy of subcritical and supercritical isobars (solid lines) and corresponding compressibility factors (dotted). Pressures from left to right $p=\{0.1,0.5,0.7,1.0,1.5,2.0,3.0\}$. All enthalpy isobars for $0<p_{r}<3$ approach a linear liquid and a linear gaseous state towards low and high temperatures, respectively. Ideal gas conditions with compressibility $Z \sim 1$ are approximately reached at the intersection of the limit lines.

The cross-over of the linear limits predicts an upper temperature bound on the existence of a transition. For argon, it occurs at approximately $360 \mathrm{~K}$, compare also to Fig. 5.

For the investigated pressure range below a reduced pressure of 3 , this point coincides with another feature: Fig. 6 also shows the compressibility factor of the isobars as a function of temperature. The compressibility factor is defined as the ratio of the actual pressure in the fluid to the pressure an ideal gas would exert at the same conditions,

$Z=p / \rho R T$,

with density $\rho$ and gas constant $R$. The compressibility factor is unity in an ideal gas and attains a different value in real fluids. Figure 6 shows that ideal gas conditions are in fact reached at the temperature of intersection of the linear limits at $360 \mathrm{~K}$, corresponding to a reduced temperature of 2.4 .

\subsection{Accuracy of the linear transition model}

The question remains as to whether the linear transition model in Eq. (6) has any practical relevance in describing real fluid transitions. As noted, its validity is limited to cases in which a liquid at subcritical or supercritical pressure is heated to a gaseous state if both start and end state are sufficiently close to the linear ideal liquid or gaseous states. On the liquid side, this is fulfilled as long as the temperature is sufficiently below the saturation temperature. On the gaseous side, Fig. 6 shows that heating beyond the cross-over temperature of the ideal asymptotes yields ideal gas compressibilities. 


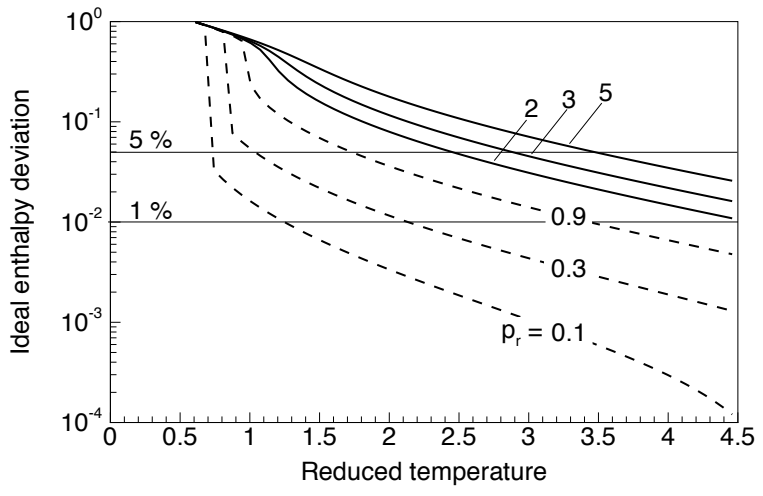

Fig. 7 Error from approximating a real fluid heating process from a $100 \mathrm{~K}$ liquid to a higher reduced temperature by the linear limit model. At a reduced pressure of 3 , heating to a reduced temperature of 3 yields a mere $5 \%$ error compared to evaluation with the NIST database. Lower pressures result in a lower required heating temperature to reach an acceptable error.

Such limitations do not restrict the model to academic cases: It is indeed relevant for processes in which the process endpoints are sufficiently far away from the transition temperature, such as in injection or combustion problems.

Figure 7 shows the difference between the linear model and an evaluation using NIST data for the illustrative case of a start temperature of $100 \mathrm{~K}$. Plotted is the error as a function of the end temperature of the heating process. For a reduced pressure of 3 , the error is $5 \%$ if the heating process reaches a reduced temperature of 3. Generally, the accuracy increases as the pressure is lowered. E.g. at a reduced pressure of 0.1 , heating to a reduced temperature of 1.2 is sufficient to reach a deviation of a mere $1 \%$.

\section{References}

[1] Nishikawa, K., Tanaka, I. "Correlation lengths and density fluctuations in supercritical states of carbon dioxide", Chemical Physics Letters, 244(1-2), pp. 149-152, 1995.

https://doi.org/10.1016/0009-2614(95)00818-O

[2] Morita, T., Nishikawa, K., Takematsu, M., Iida, H., Furutaka, S. "Structure Study of Supercritical $\mathrm{CO}_{2}$ near Higher-Order Phase Transition Line by X-ray Diffraction", The Jornal of Physical Chemistry B, 101(36), pp. 7158-7162, 1997. https://doi.org/10.1021/jp9710906

[3] Nishikawa, K., Morita, T. "Inhomogeneity of molecular distribution in supercritical fluids", Chemical Physics Letters, 316(3-4), pp. $238-242,2000$. https://doi.org/10.1016/S0009-2614(99)01241-5

[4] Santoro, M., Gorelli, F. A. "Structural changes in supercritical fluids at high pressures", Physical Review B, 77(21), p. 212103, 2008. https://doi.org/10.1103/PhysRevB.77.212103

\section{Conclusions}

An analysis of state transitions at subcritical and supercritical pressures showed that the enthalpy along isobars approaches ideal liquid and gaseous states, characterized by constant isobaric heat capacities.

These limiting states form a linear model of the enthalpy difference in liquid to gas transitions. At low pressures, this transition enthalpy is equivalent to the latent heat. As the critical pressure is approached and exceeded, a distributed latent heat becomes more and more pronounced that affects the state transition over a finite temperature interval rather than at an equilibrium saturation temperature.

This has two interesting implications: One, a property akin to latent heat is present at supercritical pressures; two, at subcritical conditions only part of the energy required for the complete state transition is supplied at equilibrium conditions in the form of the latent heat.

As a corollary, with ideal, pressure-independent end points, the heating and transition process from a densely packed ideal liquid to the perfect gas requires the same amount of energy regardless of pressure, subcritical and supercritical.

The linear transition model is furthermore useful in the analysis of processes that include the phase transition but do not have end points in the vicinity of the transition, such as injection or combustion processes. We introduced a surprisingly simple and unified way of describing the process for both subcritical and supercritical conditions.

[5] Simeoni, G. G., Bryk, T., Gorelli, F. A., Krisch, M., Ruocco, G., Santoro, M., Scopigno, T. "The Widom line as the crossover between liquid-like and gas-like behaviour in supercritical fluids", Nature Physics, 6, pp. 503-507, 2010.

https://doi.org/10.1038/nphys1683

[6] Sato, T., Sugiyama, M., Itoh, K., Mori, K., Fukunaga, T., Misawa, M., Otomo, T., Takata, S. "Structural difference between liquidlike and gaslike phases in supercritical fluid", Physical Review E, 78(5), p. 051503, 2008. https://doi.org/10.1103/PhysRevE.78.051503

[7] Brazhkin, V. V., Fomin, Y. D., Lyapin, A. G., Ryzhov, V. N., Tsiok, E. N. "Widom Line for the Liquid-Gas Transition in Lennard-Jones System", The Journal of Physical Chemistry B, 115(48), pp. 14112-14115, 2011. https://doi.org/10.1021/jp2039898 
[8] Fomin, Y. D., Ryzhov, V. N., Tsiok, E. N., Brazhkin, V. V. "Thermodynamic properties of supercritical carbon dioxide: Widom and Frenkel lines", Physical Review E, 91(2), p. 022111, 2015. https://doi.org/10.1103/PhysRevE.91.022111

[9] Fisher, M. E., Widom, B. "Decay of correlations in linear systems", The Journal of Chemical Physics, 50(9), pp. 3756-3772, 1969. https://doi.org/10.1063/1.1671624

[10] Ruppeiner, G., Sahay, A., Sarkar, T., Sengupta, G. "Thermodynamic geometry, phase transitions, and the Widom line", Physical Review E, 86(5), p. 052103, 2012. https://doi.org/10.1103/PhysRevE.86.052103

[11] Gorelli, F., Santoro, M., Scopigno, T., Krisch, M., Ruocco, G. "Liquidlike Behavior of Supercritical Fluids" Physical Review Letters, 97(24), p. 245702, 2006. https://doi.org/10.1103/PhysRevLett.97.245702

[12] McMillan, P. F., Stanley, H. E. "Going Supercritical", Nature Physics, 6, pp. 479-480, 2010. https://doi.org/10.1038/nphys1711

[13] Banuti, D. T., Raju, M., Ihme, M. "Similarity law for Widom lines and coexistence lines", Physical Review E, 95(5), p. 052120, 2017. https://doi.org/10.1103/PhysRevE.95.052120

[14] van Konynenburg, P. H., Scott, R. L., Rowlinson, J. S. "Critical lines and phase equilibria in binary van der Waals mixtures", Philosophical Transactions of the Royal Society, London. Series A, Mathematical and Physical Sciences, 298(1442), pp. 495-540, 1980. https://doi.org/10.1098/rsta.1980.0266
[15] Raju, M., Banuti, D. T., Ma, P. C., Ihme, M. "Widom Lines in Binary Mixtures of Supercritical Fluids", Scientific Reports, 7, p. $3027,2017$. https://doi.org/10.1038/s41598-017-03334-3

[16] Kafengauz, N. L., Fedorov, M. I. "Excitation of high-frequency pressure oscillations during heat exchange with diisopropylcyclohexane", Journal of Engineering Physics, 11(1), pp. 63-67, 1966. https://doi.org/10.1007/BF00829935

[17] Kafengauz, N. L., Federov, M. I. "Pseudoboiling and heat transfer in a turbulent flow", Journal of Engineering Physics, 14(5), pp. 489-490, 1968. https://doi.org/10.1007/BF00828077

[18] Banuti, D. T. "Crossing the Widom-line - Supercritical pseudo-boiling", The Journal of Supercritical Fluids, 98, pp. 12-16, 2015. https://doi.org/10.1016/j.supflu.2014.12.019

[19] Reid, R. C., Prausnitz, J. M., Poling, B. E. "The Properties of Gases and Liquids", 4th ed., McGraw Hill, New York, USA, 1987.

[20] Linstrom, P. J., Mallard, W. G. "NIST chemistry webbook, NIST Standard Reference Database Number 69", National Institute of Standards and Technology, Gaithersburg, MD, 2001 [online] Availble at: http://webbook.nist.gov/chemistry [Accessed: 17 June 2018]

[21] Oschwald, M. "Spreading angle and centerline variation of density of supercritical nitrogen jets", Atomization and Sprays, 12(1-3), pp. 91-106, 2002. https://doi.org/10.1615/AtomizSpr.v12.i123.50 